\title{
Spatial Analysis of Soil Cover by Definition of Soil Stability Indicators for Flushing in the Upper Shirvan Zone of Azerbaijan
}

\section{RAE Z H Aliyev* \\ Institute of Erosion and Irrigation NAS of Azerbaijan, Baku}

Submission: January 04, 2018; Published: January 11, 2017

*Corresponding author: RAE ZH Aliyev, Institute of Erosion and Irrigation NAS of Azerbaijan, Baku, Azerbaijan, Email: zakirakademik@mail.ru

\section{Abstract}

The results of the research prove that the "soil layer" created in the database contains information on the types of soils, as well as their groups in the southern slopes of the foothills of Azerbaijan. Given the availability of cartographic materials, an analysis was also carried out for the Akhsu district as a research facility. The results are given in Tables $1 \& 2$, which provide information on the area of specific types of soils according to altitude intervals and grades of terrain slopes.

Keywords: Light; Brown, meadow-gray; Hazard; Flushing; Carbonate; Mountain-forest; Unstable; Gray-meadow, etc

\section{Introduction}

In Aksuinsky district the largest area is occupied by bright meadow-gray soils-more than $26 \%$ of the total area. Significant area is occupied also by meadow-gray soils- $12 \%$ and graybrown-almost $11 \%$.Analyzing the distribution of soils along altitude intervals, it is established that at light up to $300 \mathrm{~m}$ above sea level, light meadow gray soils occupy $26 \%$ of the area, meadow-gray-12.3\%, and gray-brown and light gray-meadow

soils - $10 \%$.At an altitude of 300-600 m, mountain gray-brown soils occupy $4 \%$, at an altitude of $600-900 \mathrm{~m}$ - washed mountainbrown almost $7 \%$ of the total area (Table 1). Depending on the humus content of the soil and its mineral composition, the soil stability index for flushing was calculated, which was subsequently used to determine the degree of erosion threat [114].

Table 1: Distribution of soil types by altitude intervals, meters above sea level.

\begin{tabular}{|c|c|c|c|c|c|c|c|c|c|c|c|c|}
\hline \multirow{3}{*}{ Types of soil } & \multicolumn{9}{|c|}{ Altitude intervals, $\mathrm{m}$ above sea level } & \multirow{2}{*}{\multicolumn{3}{|c|}{ Together }} \\
\hline & $0-300$ & & $\begin{array}{l}300- \\
600\end{array}$ & & $\begin{array}{l}600- \\
900\end{array}$ & & $\begin{array}{l}900- \\
1200\end{array}$ & & $\begin{array}{l}1200- \\
1500\end{array}$ & & & \\
\hline & ha & $\%$ & ha & $\%$ & ha & $\%$ & ha & $\%$ & ha & $\%$ & ha & $\%$ \\
\hline $\begin{array}{l}\text { Typical mountain forest } \\
\text { brown }\end{array}$ & 0 & 0,00 & 67 & 0,07 & 682 & 0,68 & 388 & 0,39 & 104 & 0,10 & 1241 & 1,24 \\
\hline $\begin{array}{l}\text { Carbonate mountain forest } \\
\text { brown }\end{array}$ & 14 & 0,01 & 743 & 0,74 & 1510 & 1,51 & 66 & 0,07 & 0 & 0,00 & 2332 & 2,33 \\
\hline $\begin{array}{l}\text { Emerged from under } \\
\text { the forest, washed away } \\
\text { mountain brown }\end{array}$ & 36 & 0,04 & 369 & 0,37 & 1900 & 1,90 & 578 & 0,58 & 123 & 0,12 & 3006 & 3,00 \\
\hline $\begin{array}{l}\text { Emerged from under the } \\
\text { forest, carbonate mountain } \\
\text { brown }\end{array}$ & 663 & 0,66 & 1024 & 4,02 & 6710 & 6,71 & 379 & 0,38 & 0 & 0,00 & 11776 & 11,77 \\
\hline $\begin{array}{l}\text { Cultivated from under } \\
\text { forests, cultivated } \\
\text { mountain- (garden) brown }\end{array}$ & 0 & 0,00 & 0 & 0,00 & 288 & 0,29 & 89 & 0,09 & 8 & 0,01 & 385 & 0,38 \\
\hline Steppe mountain brown & 13 & 0,01 & 927 & 0,93 & 1256 & 1,26 & 5 & 0,01 & 0 & 0,00 & 2201 & 2,20 \\
\hline Mountain gray-brown & 2292 & 2,29 & 4066 & 4,06 & 337 & 0,34 & 0 & 0,00 & 0 & 0,00 & 6696 & 6,69 \\
\hline Gray-brown & 9855 & 9,85 & 781 & 0,78 & 0 & 0,00 & 0 & 0,00 & 0 & 0,00 & 10636 & 10,63 \\
\hline Light chestnut & 1092 & 1,09 & 0 & 0,00 & 0 & 0,00 & 0 & 0,00 & 0 & 0,00 & 1092 & 1,09 \\
\hline
\end{tabular}




\begin{tabular}{|c|c|c|c|c|c|c|c|c|c|c|c|c|}
\hline Light meadow gray & 26433 & 26,42 & 48 & 0,05 & 94 & 0,09 & 0 & 0,00 & 0 & 0,00 & 26575 & 26,65 \\
\hline Meadow - gray & 12308 & 12,30 & 0 & 0,00 & 0 & 0,00 & 0 & 0,00 & 0 & 0,00 & 12308 & 12,30 \\
\hline Light gray-meadow & 9983 & 9,98 & 0 & 0,00 & 0 & 0,00 & 0 & 0,00 & 0 & 0,00 & 9983 & 9,98 \\
\hline Gray meadow & 3311 & 3,31 & 0 & 0,00 & 72 & 0,07 & 0 & 0,00 & 0 & 0,00 & 3383 & 3,38 \\
\hline $\begin{array}{c}\text { Insufficiently developed } \\
\text { alluvial-meadow }\end{array}$ & 1967 & 1,97 & 10 & 0,01 & 33 & 0,03 & 0 & 0,00 & 0 & 0,00 & 2011 & 2,01 \\
\hline Swamp-meadow & 555 & 0,55 & 0 & 0,00 & 0 & 0,00 & 0 & 0,00 & 0 & 0,00 & 555 & 0,55 \\
\hline Salted & 907 & 0,91 & 0 & 0,00 & 0 & 0,00 & 0 & 0,00 & 0 & 0,00 & 907 & 0,91 \\
\hline Rest & 3543 & 3,54 & 809 & 0,81 & 571 & 0,57 & 37 & 0,04 & 0 & 0,00 & 4960 & 4,96 \\
\hline Together & 72973 & 72,94 & 11844 & 11,84 & 13452 & 13,45 & 1542 & 1,54 & 236 & 0,24 & 100047 & 100,00 \\
\hline
\end{tabular}

This indicator includes five degrees, where the 1-st stands for soils that are resistant to flushing, and the 5 th is very weakly resistant. Based on the analysis of the data in the Akhsu region, it was found that the largest area is occupied by the soils resistant to flushing (1 degree of danger) $-58 \%$ of the area as a whole. Very unstable to wash away the soil in the region is only $1.6 \%$. The largest area of soils resistant to flushing (1 degree) is in the altitude range from 0 to $300 \mathrm{~m}$. on slopes with a slope of $0-6 \%$.

Soils are 2 degrees of stability, i.e. well resistant to flushing, concentrated at an altitude of 300-800 m. with an incline of $10-18 \%$. A significant area of land belonging to this degree of stability is also at an altitude of 100-200 maslong, with a slope of $0-6 \%$. Most of the medium-resistant soils (grade 3 ) are in the altitude range of $100-300 \mathrm{~m}$. with a slope of the terrain of $0-6 \%$ (Tables 2-7).

Table 2: Distribution of soil types by grade of grade, \%.

\begin{tabular}{|c|c|c|c|c|c|c|c|c|c|c|c|c|c|c|}
\hline \multirow[t]{3}{*}{ Types of soil } & \multicolumn{11}{|c|}{ Incline ,\% } & \multirow{2}{*}{\multicolumn{3}{|c|}{ Together }} \\
\hline & \multirow{2}{*}{$\begin{array}{l}0-6 \\
\text { ha }\end{array}$} & \multirow[b]{2}{*}{$\%$} & \multirow{2}{*}{$\begin{array}{l}\text { 06- } \\
\text { Oct } \\
\text { ha } \\
\end{array}$} & \multirow[b]{2}{*}{$\%$} & \multirow{2}{*}{$\begin{array}{c}\text { Oct- } \\
18 \\
\text { ha } \\
\end{array}$} & \multirow[b]{2}{*}{$\%$} & \multirow{2}{*}{$\begin{array}{l}\text { 18- } \\
27 \\
\text { ha }\end{array}$} & \multirow[b]{2}{*}{$\%$} & \multirow{2}{*}{$\begin{array}{l}27- \\
35 \\
\text { ha }\end{array}$} & \multirow[b]{2}{*}{$\%$} & \multirow{2}{*}{$\begin{array}{l}35- \\
74 \\
\text { ha }\end{array}$} & & & \\
\hline & & & & & & & & & & & & $\%$ & ha & $\%$ \\
\hline $\begin{array}{l}\text { Typical mountain } \\
\text { forest brown }\end{array}$ & 114 & 8,51 & 265 & 19,72 & 691 & 51,42 & 197 & 14,43 & 80 & 5,93 & 0 & 0,00 & 13,44 & 1,34 \\
\hline $\begin{array}{l}\text { Carbonate mountain } \\
\text { forest brown }\end{array}$ & 138 & 5,85 & 379 & 16,12 & 1330 & 56,57 & 475 & 20,20 & 25 & 1,08 & 4 & 0,18 & 2352 & 2,35 \\
\hline $\begin{array}{l}\text { Emerged from } \\
\text { under the forest, } \\
\text { washed away } \\
\text { mountain brown }\end{array}$ & 499 & 16,90 & 1049 & 35,54 & 1291 & 43,74 & 112 & 3,79 & 1 & 0,03 & 0 & 0,00 & 2952 & 2,95 \\
\hline $\begin{array}{c}\text { Emerged from } \\
\text { under the forest, } \\
\text { carbonate mountain } \\
\text { brown }\end{array}$ & 1395 & 11,70 & 2313 & 19,41 & 6495 & 54,50 & 1688 & 14,16 & 26 & 0,22 & 0 & 0,00 & 11916 & 11,91 \\
\hline $\begin{array}{c}\text { Cultivated from } \\
\text { under forests, } \\
\text { cultivated } \\
\text { mountain- (garden) } \\
\text { brown }\end{array}$ & 78 & 20,14 & 163 & 42,21 & 133 & 34,47 & 12 & 3,18 & 0 & 0,00 & 0 & 0,00 & 385 & 0,38 \\
\hline $\begin{array}{l}\text { Steppe mountain } \\
\text { brown }\end{array}$ & 52 & 2,37 & 207 & 9,42 & 1261 & 57,40 & 675 & 30,75 & 1 & 0,05 & 0 & 0,00 & 2196 & 2,20 \\
\hline $\begin{array}{l}\text { Mountain gray- } \\
\text { brown }\end{array}$ & 439 & 6,75 & 1384 & 21,28 & 3976 & 61,11 & 689 & 10,60 & 17 & 0,00 & 0 & 0,00 & 6506 & 6,50 \\
\hline Gray-brown & 7897 & 74,11 & 1074 & 10,08 & 1342 & 12,60 & 337 & 3,16 & 6 & 0,00 & 0 & 0,00 & 10656 & 10,65 \\
\hline Light chestnut & 1018 & 93,21 & 42 & 3,82 & 32 & 2,97 & 0 & 0,00 & 0 & 0,00 & 0 & 0,00 & 1092 & 1,09 \\
\hline Light meadow gray & 26232 & 99,08 & 99 & 0,37 & 139 & 0,53 & 5 & 0,02 & 0 & 0,00 & 0 & 0,00 & 26475 & 26,49 \\
\hline Meadow - gray & 12366 & 99,98 & 3 & 0,02 & 0 & 0,00 & 0 & 0,00 & 0 & 0,00 & 0 & 0,00 & 12368 & 12,36 \\
\hline Light gray-meadow & 99561 & 99,67 & 14 & 0,14 & 18 & 0,18 & 0 & 0,00 & 0 & 0,00 & 0 & 0,00 & 9983 & 9,98 \\
\hline Gray meadow & 3320 & 98,15 & 40 & 1,20 & 22 & 0,65 & 0 & 0,00 & 0 & 0,00 & 0 & 0,00 & 3383 & 3,38 \\
\hline
\end{tabular}




\section{Global Journal of Otolaryngology}

\begin{tabular}{|c|c|c|c|c|c|c|c|c|c|c|c|c|c|c|}
\hline $\begin{array}{c}\text { Insufficiently } \\
\text { developed alluvial- } \\
\text { meadow }\end{array}$ & 1950 & 96,99 & 8 & 0,42 & 41 & 2,02 & 12 & 0,57 & 0 & 0,00 & 0 & 0,00 & 2011 & 2,10 \\
\hline Swamp-meadow & 555 & 100,00 & 0 & 0,00 & 0 & 0,00 & 0 & 0,00 & 0 & 0,00 & 0 & 0,00 & 555 & 0,55 \\
\hline Salted & 907 & 100,0 & 0 & 0,00 & 0 & 0,00 & 0 & 0,00 & 0 & 0,00 & 0 & 0,00 & 907 & 0,91 \\
\hline Rest & 4035 & 81,35 & 374 & 7,53 & 421 & 8,49 & 126 & 2,54 & 0 & 0,00 & 0 & 0,00 & 4960 & 4,96 \\
\hline Together & 70944 & 70,92 & 7413 & 7,41 & 17193 & 17,19 & 4326 & 4,32 & 160 & 0,16 & 4 & 0,00 & 100040 & 100,00 \\
\hline
\end{tabular}

Table 3: Area (ha and \%) of soils belonging to 1 degree of resistance to flushing.

\begin{tabular}{|c|c|c|c|c|c|c|c|c|c|c|c|c|}
\hline \multirow{3}{*}{ Height m. } & \multicolumn{10}{|c|}{ Slope,\% } & \multirow{2}{*}{\multicolumn{2}{|c|}{ Together }} \\
\hline & \multirow{2}{*}{$\begin{array}{l}0-6 \\
\text { ha }\end{array}$} & \multirow[b]{2}{*}{$\%$} & \multirow{2}{*}{$\begin{array}{c}6-10 \\
\text { ha }\end{array}$} & \multirow[b]{2}{*}{$\%$} & \multirow{2}{*}{$\begin{array}{c}\text { 10-18 } \\
\text { ha }\end{array}$} & \multirow[b]{2}{*}{$\%$} & \multirow{2}{*}{$\begin{array}{c}\text { 18-27 } \\
\text { ha }\end{array}$} & \multirow[b]{2}{*}{$\%$} & \multicolumn{2}{|c|}{$27-37$} & & \\
\hline & & & & & & & & & ha & $\%$ & ha & $\%$ \\
\hline $0-100$ & 25103 & 42,32 & 16 & 0,03 & 13 & 0,02 & 0 & 0,00 & 0 & 0,00 & 25133 & 42,37 \\
\hline $100-200$ & 7790 & 13,13 & 337 & 0,57 & 129 & 0,22 & 18 & 0,03 & 0 & 0,00 & 8274 & 13,95 \\
\hline $200-300$ & 7790 & 13,13 & 337 & 0,57 & 129 & 0,22 & 18 & 0,03 & 0 & 0,00 & 8274 & 13,95 \\
\hline $300-400$ & 869 & 1,47 & 624 & 1,05 & 619 & 1,04 & 74 & 0,12 & 0 & 0,00 & 2187 & 3,69 \\
\hline $400-500$ & 56 & 0,09 & 236 & 0,40 & 1217 & 2,05 & 483 & 0,81 & 5 & 0,01 & 1997 & 3,37 \\
\hline $500-600$ & 68 & 0,11 & 317 & 0,53 & 1532 & 2,58 & 572 & 0,96 & 4 & 0,01 & 2492 & 4,20 \\
\hline $600-700$ & 176 & 0,30 & 533 & 0,90 & 2210 & 3,73 & 479 & 0,81 & 19 & 0,03 & 3418 & 5,76 \\
\hline $700-800$ & 514 & 0,87 & 1215 & 2,05 & 1946 & 3,28 & 203 & 0,34 & 16 & 0,03 & 3893 & 6,56 \\
\hline $800-900$ & 547 & 0,92 & 801 & 1,35 & 811 & 1,37 & 91 & 0,15 & 9 & 0,02 & 2259 & 3,81 \\
\hline $900-1000$ & 122 & 0,21 & 247 & 0,42 & 200 & 0,34 & 41 & 0,07 & 8 & 0,01 & 618 & 1,04 \\
\hline $1000-1100$ & 20 & 0,03 & 174 & 0,29 & 236 & 0,40 & 19 & 0,03 & 7 & 0,01 & 456 & 0,77 \\
\hline $1100-1200$ & 19 & 0,03 & 31 & 0,05 & 111 & 0,19 & 27 & 0,04 & 3 & 0,00 & 190 & 0,32 \\
\hline $1200-1300$ & 0 & 0,00 & 6 & 0,01 & 60 & 0,10 & 27 & 0,05 & 2 & 0,00 & 94 & 0,16 \\
\hline $1300-1400$ & 0 & 0,00 & 3 & 0,01 & 16 & 0,03 & 4 & 0,01 & 1 & 0,00 & 24 & 0,04 \\
\hline Together & 43074 & 72,63 & 4878 & 8,22 & 9229 & 15,56 & 2055 & 3,46 & 75 & 0,13 & 59310 & 100,00 \\
\hline
\end{tabular}

Table 4 : Area (ha and \%) of soils belonging to the 2 nd degree of resistance to flushing.

\begin{tabular}{|c|c|c|c|c|c|c|c|c|c|c|c|c|}
\hline \multirow{3}{*}{$\begin{array}{l}\text { Height } \\
\text { m. }\end{array}$} & \multicolumn{10}{|c|}{ Slope,\% } & \multirow{2}{*}{\multicolumn{2}{|c|}{ Together }} \\
\hline & \multicolumn{2}{|c|}{$0-6$} & \multicolumn{2}{|c|}{$06-10$} & \multicolumn{2}{|c|}{$10-18$} & \multicolumn{2}{|c|}{$18-27$} & \multicolumn{2}{|c|}{$27-37$} & & \\
\hline & ha & $\%$ & ha & $\%$ & ha & $\%$ & ha & $\%$ & ha & $\%$ & ha & $\%$ \\
\hline $0-100$ & 2 & 0,02 & 0 & 0,00 & 0 & 0,00 & 0 & 0,00 & 0 & 0,00 & 2 & 0,02 \\
\hline $100-200$ & 710 & 8,43 & 148 & 1,76 & 123 & 1,47 & 10 & 0,12 & 0 & 0,00 & 992 & 11,78 \\
\hline $200-300$ & 239 & 2,84 & 243 & 2,89 & 683 & 8,11 & 69 & 0,82 & 2 & 0,02 & 1237 & 14,68 \\
\hline $300-400$ & 61 & 0,73 & 232 & 2,75 & 775 & 9,20 & 183 & 2,18 & 11 & 0,13 & 1263 & 14,99 \\
\hline $400-500$ & 39 & 0,46 & 84 & 0,99 & 689 & 8,18 & 256 & 3,04 & 8 & 0,09 & 1075 & 12,76 \\
\hline $500-600$ & 38 & 0,45 & 119 & 1,41 & 732 & 8,69 & 321 & 3,81 & 2 & 0,02 & 1211 & 14,37 \\
\hline $600-700$ & 21 & 0,24 & 146 & 1,74 & 863 & 10,23 & 331 & 3,92 & 0 & 0,00 & 1360 & 16,14 \\
\hline $700-800$ & 52 & 0,61 & 155 & 1,84 & 538 & 6,39 & 169 & 2,01 & 6 & 0,07 & 920 & 10,92 \\
\hline $800-900$ & 31 & 0,36 & 59 & 0,70 & 150 & 1,78 & 55 & 0,65 & 3 & 0,04 & 297 & 3,53 \\
\hline
\end{tabular}




\begin{tabular}{|c|c|c|c|c|c|c|c|c|c|c|c|c|}
\hline $\begin{array}{l}900- \\
1000\end{array}$ & 3 & 0,04 & 11 & 0,13 & 27 & 0,32 & 19 & 0,23 & 4 & 0,05 & 64 & 0,76 \\
\hline $\begin{array}{c}1000- \\
1100\end{array}$ & 0 & 0,00 & 0 & 0,00 & 4 & 0,04 & 1 & 0,01 & 0 & 0,00 & 4 & 0,05 \\
\hline Together & 1194 & 14,18 & 1197 & 14,21 & 4583 & 54,40 & 1414 & 16,79 & 36 & 0,43 & 8425 & 100,00 \\
\hline
\end{tabular}

Table 5: Area (ha and \%) of soils belonging to the 3 degree of resistance to flushing.

\begin{tabular}{|c|c|c|c|c|c|c|c|c|c|c|c|c|}
\hline \multirow{3}{*}{$\begin{array}{l}\text { Height } \\
\text { m. }\end{array}$} & \multicolumn{10}{|c|}{ Slope, $\%$} & \multirow{2}{*}{\multicolumn{2}{|c|}{ Together }} \\
\hline & \multicolumn{2}{|c|}{$0-6$} & \multicolumn{2}{|c|}{ 06-0ct } & \multicolumn{2}{|c|}{ Oct-18 } & \multicolumn{2}{|c|}{$18-27$} & \multicolumn{2}{|c|}{$27-37$} & & \\
\hline & га & $\%$ & га & $\%$ & га & $\%$ & га & $\%$ & га & $\%$ & га & $\%$ \\
\hline $0-100$ & 23051 & 78,78 & 0 & 0,00 & 0 & 0,00 & 0 & 0,00 & 0 & 0,00 & 23052 & 78,78 \\
\hline $100-200$ & 2100 & 7,18 & 261 & 0,89 & 158 & 0,54 & 30 & 0,10 & 0 & 0,00 & 2549 & 8,71 \\
\hline $200-300$ & 1557 & 5,32 & 195 & 0,67 & 379 & 1,30 & 136 & 0,46 & 1 & 0,00 & 2268 & 7,75 \\
\hline $300-400$ & 34 & 0,12 & 42 & 0,14 & 165 & 0,56 & 41 & 0,14 & 4 & 0,01 & 287 & 0,98 \\
\hline $400-500$ & 7 & 0,02 & 44 & 0,15 & 278 & 0,95 & 85 & 0,29 & 1 & 0,00 & 414 & 1,41 \\
\hline $500-600$ & 13 & 0,04 & 53 & ,18 & 273 & 0,93 & 74 & 0,25 & 0 & 0,00 & 413 & 1,41 \\
\hline $600-700$ & 3 & 0,01 & 23 & 0,08 & 141 & 0,48 & 41 & 0,14 & 0 & 0,00 & 209 & 0,71 \\
\hline $700-800$ & 4 & 0,01 & 28 & 0,10 & 23 & 0,08 & 3 & 0,01 & 0 & 0,00 & 58 & 0,20 \\
\hline $800-900$ & 0 & 0,00 & 10 & 0,03 & 1 & 0,00 & 0 & 0,00 & 0 & 0,00 & 10 & 0,04 \\
\hline Together & 26770 & 91,49 & 658 & 2,25 & 1417 & 4,84 & 409 & 0,40 & 5 & 0,02 & 29259 & 100,00 \\
\hline
\end{tabular}

Table 6: Area (ha and\%) of soils belonging to the 4 degree of resistance to flushing .

\begin{tabular}{|c|c|c|c|c|c|c|c|c|c|c|c|c|}
\hline \multirow{3}{*}{$\begin{array}{c}\text { Height } \\
\text { m. }\end{array}$} & \multicolumn{10}{|c|}{ Slope,\% } & \multirow{2}{*}{\multicolumn{2}{|c|}{ Together }} \\
\hline & \multicolumn{2}{|c|}{$0-6$} & \multicolumn{2}{|c|}{$6-10$} & \multicolumn{2}{|c|}{$10-18$} & \multicolumn{2}{|c|}{$18-27$} & \multicolumn{2}{|c|}{$27-37$} & & \\
\hline & ha & $\%$ & ha & $\%$ & ha & $\%$ & ha & $\%$ & ha & $\%$ & ha & $\%$ \\
\hline $0-100$ & 2285 & 57,36 & 0 & 0,00 & 0 & 0,00 & 0 & 0,00 & 0 & 0,00 & 2285 & 57,36 \\
\hline $100-200$ & 54 & 13,55 & 27 & 0,69 & 60 & 1,51 & 2 & 0,05 & 0 & 0,00 & 630 & 15,80 \\
\hline $200-300$ & 61 & 1,52 & 32 & 0,82 & 93 & 2,34 & 20 & 0,51 & 0 & 0,0 & 207 & 5,19 \\
\hline $300-400$ & 0 & 0,01 & 3 & 0,7 & 17 & 0,42 & 9 & 0,24 & 0 & 0,00 & 29 & 0,74 \\
\hline $400-500$ & 0 & 0,00 & 0 & 0,00 & 0 & 0,00 & 5 & 0,12 & 0 & 0,00 & 5 & 0,12 \\
\hline $500-600$ & 0 & 0,00 & 3 & 0,07 & 28 & 0,71 & 5 & 0,11 & 0 & 0,00 & 36 & 0,89 \\
\hline $600-700$ & 5 & 0,13 & 6 & 0,14 & 78 & 1,95 & 22 & 0,56 & 0 & 0,00 & 111 & 2,79 \\
\hline $700-800$ & 24 & 0,60 & 73 & 1,83 & 131 & 3,30 & 29 & 0,74 & 1 & 0,02 & 258 & 6,48 \\
\hline $800-900$ & 17 & 0,43 & 80 & 2,00 & 74 & 1,83 & 17 & 0,42 & 1 & 0,03 & 189 & 4,74 \\
\hline $900-1000$ & 22 & 0,55 & 10 & 0,24 & 8 & 0,21 & 20 & 0,49 & 3 & 0,09 & 63 & 1,58 \\
\hline $\begin{array}{c}1000- \\
1100\end{array}$ & 8 & 0,21 & 20 & 0,25 & 39 & 0,97 & 8 & 0,19 & 9 & 0,22 & 83 & 2,09 \\
\hline $\begin{array}{l}1100- \\
1200 \\
\end{array}$ & 4 & 0,10 & 5 & 0,12 & 16 & 0,40 & 6 & 0,14 & 15 & 0,37 & 46 & 1,14 \\
\hline $\begin{array}{c}1200- \\
1300\end{array}$ & 0 & 0,00 & 1 & 0,02 & 22 & 0,56 & 2 & 0,06 & 11 & 0,27 & 36 & 0,91 \\
\hline $\begin{array}{l}1300- \\
1400 \\
\end{array}$ & 0 & 0,00 & 1 & 0,01 & 4 & 0,10 & 1 & 0,02 & 2 & 0,04 & 7 & 0,17 \\
\hline Together & 2966 & 74,45 & 260 & 6,53 & 571 & 14,34 & 145 & 3,65 & 41 & 1,03 & 3984 & 100,00 \\
\hline
\end{tabular}


Table 7: Area (ha and \%) of soils belonging to the 5 degree of resistance to flushing.

\begin{tabular}{|c|c|c|c|c|c|c|c|c|c|c|c|c|}
\hline \multirow{3}{*}{$\begin{array}{c}\text { Height } \\
\text { m. }\end{array}$} & \multicolumn{10}{|c|}{ Slope,\% } & \multirow{2}{*}{\multicolumn{2}{|c|}{ Together }} \\
\hline & \multicolumn{2}{|c|}{$0-6$} & \multicolumn{2}{|c|}{ 06-0ct } & \multicolumn{2}{|c|}{ Oct-18 } & \multicolumn{2}{|c|}{$18-27$} & \multicolumn{2}{|c|}{$27-37$} & & \\
\hline & ha & $\%$ & ha & $\%$ & ha & $\%$ & ha & $\%$ & ha & $\%$ & ha & $\%$ \\
\hline $0-100$ & 199 & 12,03 & 0 & 0,00 & 0 & 0,00 & 0 & 0,00 & 0 & 0,00 & 199 & 1203 \\
\hline $100-200$ & 113 & 6,82 & 7 & 0,41 & 10 & 0,59 & 15 & 0,88 & 0 & 0,00 & 144 & 8,70 \\
\hline $200-300$ & 351 & 21,19 & 21 & 1,26 & 44 & 2,69 & 17 & 1,03 & 2 & 0,13 & 436 & 26,30 \\
\hline $300-400$ & 105 & 6,35 & 27 & 1,61 & 48 & 2,88 & 33 & 2,02 & 2 & 0,09 & 214 & 12,94 \\
\hline $400-500$ & 113 & 6,84 & 26 & 1,59 & 37 & 2,22 & 5 & 0,29 & 0 & 0,00 & 181 & 10,95 \\
\hline $500-600$ & 154 & 9,28 & 46 & 2,79 & 30 & 1,78 & 3 & 0,19 & 0 & 0,00 & 233 & 14,04 \\
\hline $600-700$ & 113 & 6,85 & 41 & 2,47 & 43 & 2,62 & 1 & 0,06 & 0 & 0,01 & 199 & 12,01 \\
\hline $700-800$ & 45 & 2,70 & 3 & 0,17 & 3 & 0,15 & 0 & 0,00 & 0 & 0,00 & 50 & 3,02 \\
\hline Together & 1194 & 72,05 & 171 & 10,30 & 214 & 12,94 & 74 & 4,48 & 4 & 0,23 & 1657 & 100,00 \\
\hline
\end{tabular}

Weakly resistant soils to flushing ( $4^{\text {th }}$ degree) occupy almost $71 \%$ of the area in the altitude range from 100 to $200 \mathrm{~m}$. with a slope of $0-6 \%$. Most of the soils are very unstable to flushing (grade 5) - more than 69\%, are located on slopes with a slight slope of $0-6 \%$ and a height of 100 to $700 \mathrm{~m}$.

\section{References}

1. Alekperov K A (1980) Soil-erosion map and land protection. Moscow, Russia.

2. Aliev B H (2005) The problem of desertification in Azerbaijan and ways to solve it. Ziya-Nurlan Publishing House. Baku, Azerbaijan.

3. Aliev B H, Aliev Z H, Aliev I N (2005) Problems of erosion in Azerbaijan and ways to solve it. "Ziya-NIC" Nurlan "Publishing House, Baku: 122.

4. Ibragimov A A Mapping of eroded soils on agricultural land (on the example of the Dashkesan region of the Azerbaijan SSR) Questions of the methodology of soil-erosion mapping. Moscow, Russia.

5. The decree of the Ministry of Agriculture of the Republic of Poland and the development of the village dated 11.03 .2009 on the details of the terms and procedures for the provision of financial assistance in the framework of the works "Support to management in mountainous areas and other areas with unpleasant conditions of subsistence" ("NUKH"), covered by the "Program development of rural areas in 2007-2013 “. Bulletin of Laws 68: 448.

6. Order of the Council (European Commonwealth) 1698/2005 of 20.09.2005 On the issue of supporting the development of rural areas by the "European Agricultural Fund" in the field of "Rural Development" ("Bulletin of the laws of the U.S. of October 21, 2005)

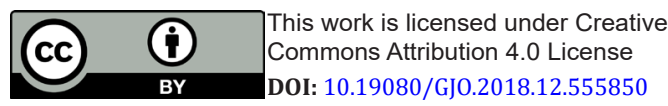

7. Order of the Council (European Commonwealth) No.77 / 2009 of 19.01.2009. Establishing general rules of the system of direct support of farmers in the field of common agricultural policy and establishing a certain system of support to farmers, changing order (EC) No. 1290/2005, (EU) №247 / 2006, (EU) №378 / 2007 and rejecting the order (EC) No.1782 / 2003 ("Law bulletin" EU L-30 "dated January 31, 2009, page 16)

8. Commission Regulation (EC) No 1974/2006 of 15.12.2006, which defines the detailed rules for the application of the Council of the European Union No. 1698/2005 on supporting the development of rural areas by the "European Agricultural Fund" in the field of "Rural Development" ("RSR"). ("Bulletin of Laws") of the EU "L 368" of December 23, 2006, p. 15, with the trace of the amendment)

9. The Law of 07.03.2007 on Supporting Rural Development with the participation of the "European Agricultural Fund" in the field of "Rural Development"; 64, pos.427

10. www.minrol.gov.pl

11. www/armir.gov.pl

12. www.mos.gov.pl

13. Www.polskapomoc.gov.pl. Training materials ISBN 978-83-61875-147. Practical course. Minikovo.14-18.09.2009. Falent. Poland 2009

14. www.Project 640 / 2010. Principles for the formation of the structure of agricultural lands in mountain regions of Azerbaijan subject to erosion, using GIS technology. Training materials ISBN 978-83-6241610-3. Falent Poland 2010.

\section{Your next submission with Juniper Publishers will reach you the below assets}

- Quality Editorial service

- Swift Peer Review

- Reprints availability

- E-prints Service

- Manuscript Podcast for convenient understanding

- Global attainment for your research

- Manuscript accessibility in different formats

( Pdf, E-pub, Full Text, Audio)

- Unceasing customer service

Track the below URL for one-step submission https://juniperpublishers.com/online-submission.php 